\title{
Single vaginal dose of $800 \mu g$ misoprostol for termination of first trimester spontaneous incomplete or missed miscarriage
}

\begin{abstract}
Background: Vaginal misoprostol have been used for of first trimester miscarriage. But studies are lacking an ideal effective dose, frequency and patient's satisfaction. This study was done to evaluate the effectiveness and side effects of a single vaginal dose of $800 \mu \mathrm{g}$ misoprostol for termination of first trimester miscarriage.
\end{abstract}

Materials and methods: This study was conducted in the Women specialized hospital, King Fahad Medical City, Riyadh, Kingdom of Saudi Arabia from October 2010 to May 2012. A prospective study was conducted for 100 pregnant women who were admitted to Women Specialized Hospital, Riyadh, with spontaneous incomplete or missed miscarriage. All admitted patients were given $800 \mu \mathrm{g}$ of misoprostol as a single vaginal dose. Twentyfour hours after the first dose, patients were evaluated for whether they had a complete miscarriage, went for dilatation and curettage $(\mathrm{D} \& \mathrm{C})$ or needed a second repeat dose. The mean time from induction to miscarriage, vaginal loss, side effects, and complications were evaluated.

Results: A total of 50 patients $(50 \%)$ had aborted completely after the first dose within the first 24 hours and 5 patients $(5 \%)$ after the second dose. The mean time to complete miscarriage after the first dose was $7 \pm 4.2$ hours. The remaining 45 patients $(45 \%)$ went for emergency $\mathrm{D} \& \mathrm{C}$ due to excessive bleeding and/or the gestational sac not being passed. There were significant reductions in pulse rate, systolic blood pressure and hemoglobin level post misoprostol administration. However, there was no significant difference in diastolic blood pressure and patients were satisfied.

Conclusion: Vaginal route misoprostol can be used safely to terminate first trimester spontaneous incomplete or missed miscarriages with acceptable rates of success and minimal side effects.

Keywords: misoprostol, termination of pregnancy, early pregnancy failure
Volume 2 Issue 3 - 2015

\author{
Dania H Al-Jaroudi, ${ }^{2}$ Nora Al-Banyan,' \\ Ahmed Saleh, ${ }^{2}$ Salih Al Salih ${ }^{3}$ \\ 'Pharmacy Services Administration, Clinical Pharmacist, Saudi \\ Arabia \\ ${ }^{2}$ Reproductive Endocrinology and Infertility Medicine \\ Department (REIMD), Womens Specialized Hospital, Saudi \\ Arabia \\ ${ }^{3}$ Consultant Obstetrics and Gynecology, Saudi British Hospital, \\ Saudi Arabia
}

\section{Correspondence: Dania H Al-Jaroudi, Consultant} Obstetrics and Gynecology, Assistant Professor, Reproductive Endocrinology and Infertility, Minimally Invasive Gynecologic Surgery, Reproductive Endocrinology and Infertility Medicine Department (REIMD),Women's Specialized Hospital, King Fahad Medical City, PO Box 59046, Riyadh I I525, Saudi Arabia, Tel 966-I-288-9999, Email daljaroudi@kfmc.med.sa

Received: April II, 2015 | Published: June 03, 2015

\section{Introduction}

Spontaneous miscarriage occurs in $15-20 \%$ of all clinically recognized pregnancies, of which $80 \%$ occur in the first trimester and $20 \%$ occur in the second trimester. ${ }^{1}$ Incomplete and missed miscarriages can be managed expectantly, surgically, or with medical therapy. ${ }^{2-4}$ Expectant management works well and is convenient for some patients. However, it is more costly and less desirable to women who prefer immediate treatment. ${ }^{4}$ For pregnancies of less than 14 weeks gestation, surgical evacuation by dilatation and curettage $(D \& C)$ is the most commonly performed standard procedure. ${ }^{5}$ However, complications arise such as cervical injury, uterine perforation, lifethreatening hemorrhage, post-surgical pelvic infection and anesthetic complications. ${ }^{6,7}$ Recently, the use of Prostaglandins for termination of first trimester miscarriage has become a safe, effective and less invasive alternative procedure. ${ }^{6}$ Prostaglandin E2 has been commonly used by many obstetricians for pregnancy termination, however, due to its high cost, its usage has declined. Synthetic Prostaglandin E1 (Misoprostol), a cheaper alternative medication, has also been effectively used for first and second trimester miscarriage termination. ${ }^{8,9}$ Misoprostol also known as Cytotec ${ }^{\circledR}$ has been approved by the US FDA for the prevention and treatment of gastric ulcers. ${ }^{8}$ Misoprostol was first used to induce abortion in Brazil by Barbosa \& Arilha. ${ }^{10}$ Following this discovery, several studies have proven its safety, acceptability and effectiveness for miscarriage termination with success rates of $40-90 \%$ versus $30-40 \%$ with expectant management. ${ }^{11,12}$ Several misoprostol dosages (200-800 $\mu \mathrm{g})$, regimens, settings (inpatient versus outpatient), routes (oral versus vaginal) and indications (incomplete or missed miscarriage) have been used to treat early pregnancy miscarriages. ${ }^{11-15}$ A Cochrane review included 19 randomized controlled trials on pregnancies less than 14 weeks and concluded that vaginal misoprostol shortens the time to expulsion when compared with placebo. ${ }^{16}$ Furthermore, recent randomized studies have shown that vaginal misoprostol of doses $400-800 \mu \mathrm{g}$ is highly effective, takes less time to expulsion and has less side effects than similar doses given orally. ${ }^{13-16}$ These studies on vaginal misoprostol for treatment of spontaneous first trimester pregnancy failure are still lacking an ideal profile for effective dose and frequency with acceptable side effects. The present prospective study was undertaken to evaluate the effectiveness, side effects and patient's satisfaction among inpatients who received a single vaginal dose of $800 \mu \mathrm{g}$ as management for first trimester spontaneous incomplete and missed miscarriages.

\section{Materials and methods}

This prospective clinical study was conducted in the Women Specialized Hospital, King Fahad Medical City, Riyadh, Kingdom of 
Saudi Arabia from October 2010 to May 2012. The study protocol was approved by the hospital Institutional Review Board. All patients who were admitted to the hospital for the management of incomplete or missed miscarriages were offered and consented to participate in the study protocol. The diagnosis was made after history evaluation, physical examination, and serial transvaginal ultrasound (TVS) and serial $\beta$-HCG levels. All women aged $18-40$ years old, with a diagnosis of blighted ovum, missed or in complete miscarriage, between 6 and 13 weeks gestation and who agreed to participate were included in the study. Patients were excluded from the study if they had a history of prostaglandins allergy, asthma, hypertension, heart disease, renal or liver dysfunction, hemodynamic instability, septic miscarriage, ectopic pregnancy or trophoblastic disease. A total of 100 pregnant women who fulfilled the inclusion criteria were enrolled in the study. The indications for miscarriage termination were incomplete miscarriage $(\mathrm{n}=42)$, absence of an embryo/fetus cardiac activity $(\mathrm{n}=45)$ and an empty gestational sac (blighted ovum) $(\mathrm{n}=13)$. Patient demographic characteristics are depicted in Table 1. Clinical parameters, pule rate, systolic and diastolic blood pressure and temperature were recorded before and after misoprostol administration (Table 2). Gestational age was calculated from the first day of last menstrual cycle, pelvic examination and ultrasonogram assessment. All patients on admission had laboratory investigations, complete blood count, blood group and rhesus status, and coagulation profile. Patients were restricted nothing per oral and intravenous fluid was administered.

Table I Demographic characteristics of the study population $(n=100)$

\begin{tabular}{ll}
\hline \multicolumn{2}{l}{ Demographic data } \\
\hline Age (year) & $30.6 \pm 6.6$ \\
Nulliparous & $34(34)$ \\
Multiparous & $66(66)$ \\
Gestational Age (week) & $10 \pm 2.5$ \\
\hline
\end{tabular}

"Values are means \pm SD with (\%) in parentheses.

\section{Treatment protocol}

All included patients received $800 \mu \mathrm{g}$ of misoprostol (Cytotec $200 \mu \mathrm{g}$, Searle, High Wycombe, England) as a single vaginal dose consisting of four tablets of $200 \mu \mathrm{g}$ each. After inserting vaginal misoprostol in the posterior fornix, the patients were restricted to bed rest for 40 minutes and their vital signs, vaginal loss, side effects and complications were recorded at 2 hour intervals during the first 24 hours and after abortion. No additional co-interventions were used. Patients would receive intramuscular or intravenous narcotic agents for pain control. If side effects such as fever occurred, patients would receive acetaminophen $500-1,000 \mathrm{mg}$ orally. Success was defined as complete evacuation of the uterus without recourse to surgical intervention. Missed abortion was defined as ultrasound evidence of intact empty gestational sac or an embryo/fetus with no evidence of fetal cardiac activity and closed cervical os. In cases of complete abortion, patients were assessed by clinical examination and the findings were confirmed by pelvic sonogram. In cases of incomplete abortion with excessive vaginal bleeding, emergency D\&C was performed. In cases of incomplete abortion without excessive vaginal bleeding, patients were offered a second repeat dose or D\&C. If complete abortion had not occurred or the gestational sac was still found 12 hours after the second dose, these cases were considered failure cases and patients were taken for D\&C. Side effects such as pain, nausea, vomiting, fever, diarrhea, and prolonged and/or heavy bleeding were recorded and evaluated. The patients who had excessive bleeding and Hct $\leq 30 \%$ were considered for blood transfusion. The initiation of abortion and the time interval from vaginal misoprostol insertion to complete expulsion of conceptus were evaluated and recorded by the on call obstetrician. All Rh-negative patients were given anti-D immuno prophylaxis after miscarriage before discharge. A brief questionnaires were given to all patients during and after the abortion in order to assess their experience and satisfaction with the medical regimen.

\section{Data analyses}

Statistical comparisons were performed with descriptive techniques and two-tailed $t$ tests were used for continuous data. A paired t-test was used for comparison of pulse rate, systolic and diastolic blood pressure and temperature before and after misoprostol administration. Fisher's exact and chi-square tests were used for categorical data and a $\mathrm{P}$ value $<0.05$ was considered statistically significant.

\section{Results}

A total of 100 women completed the study and were eligible for final analysis. A total of 50 patients (50\%) had aborted completely after the first vaginal dose within the first 24 hours and 5 patients $(5 \%)$ after the second dose. The mean time to complete miscarriage after the first dose was $7 \pm 4.2$ hours. There was no significant difference between patients who aborted following the first dose with a diagnosis of missed miscarriage $(n=30)$ versus incomplete miscarriage $(n=20)$ $\mathrm{P}=0.07$, 95\% CI: $0.9-5.4$. A total of 38 patients (38\%) went for emergency $\mathrm{D} \& \mathrm{C}$ due to excessive bleeding following the first misoprostol dose and 7 patients (7\%) went for D \& C after the second dose due to mild bleeding and due to the gestational sac not being passed. There were significant reductions in pulse rate, systolic blood pressure and hemoglobin level post misoprostol administration (Table 2). However, there was no significant difference in diastolic blood pressure (Table 2). There was a significant increase in temperature post misoprostol administration (Table 2). Side effects occurring within the first 24 hours are shown in Table 3. No uterine rupture was reported in our study and no patients required blood transfusion. Seventy one patients reported that they were satisfied with the medical treatment and regimen, and 29 patients reported they were unsatisfied (71\% vs $29 \%)(\mathrm{P}<0.001,95 \%$ CI: 3.1-11.6)

Table 2 Comparing pre and post misoprostol parameters

\begin{tabular}{llll}
\hline Parameter & Pre Misoprostol & Post Misoprostol & PValue \\
\hline Pulse per Minute & $84 \pm 10.8$ & $81 \pm 11.2$ & 0.01 \\
Temperature $\left({ }^{\circ} \mathrm{C}\right)$ & $36.9 \pm 0.5$ & $37.1 \pm 0.5$ & 0.001 \\
Systolic Blood Pressure $(\mathrm{mmHg})$ & $112 \pm 11.0$ & $110 \pm 10.8$ & 0.04 \\
Diastolic Blood Pressure $(\mathrm{mmHg})$ & $66 \pm 7.0$ & $65 \pm 7.0$ & 0.1 \\
Hemoglobin $(\mathrm{g} / \mathrm{dl})$ & $12.3 \pm 1.3$ & $11.4 \pm 1.2$ & 0.001
\end{tabular}


Table 3 Side effects encountered within the first 48 hours $(n=22 / 100)$

\begin{tabular}{ll}
\hline Side effects & $\mathbf{N}(\%)$ \\
\hline Nausea/Vomiting & $13(13)$ \\
Fever & $6(6)$ \\
Bleeding & $3(3)$ \\
Total & $22(22)$ \\
\hline
\end{tabular}

\section{Discussion}

Misoprostol (Cytotec), a synthetic analog of prostaglandin E1, has been proven to be an effective, safe and cheap alternative to surgical evacuation, with varying adverse events and success rates. It can be given in both outpatient and inpatient settings. In this study we prospectively reviewed 100 patients who were diagnosed with incomplete or missed miscarriage at $<14$ weeks' gestation. All patients who were admitted to the hospital had a single vaginal misoprostol dose of $800 \mu \mathrm{g}$ alone or with a repeat dose after 24 hours for a maximum of two doses (total of $1600 \mu \mathrm{g}$ ). We had a total success rate (complete miscarriage) of $55 \%$, within 48 hours. The remaining 45 patients who required D\&C were considered failure cases with a rate of $45 \%$. Our success rate of $55 \%$ is lower than previously reported success rates described by other studies of $60-90 \%$, but similar to Ho PC et al. ${ }^{12}$ who reported success rate of $50 \%$ using the vaginal route. ${ }^{12}$ This is due to the shorter observation time following misoprostol administration for the first and the second doses (24-48 hours). Other studies used expectant times between 2 to 7 days before giving the second dose. Their higher success rates were probably due to more observation times being given to allow for complete spontaneous uterine expulsion and/or more frequent misoprostol doses. However, Ngoc et al. ${ }^{17}$ reported in a randomized study that their mean time from induction to expulsion was significantly longer than our study $(13.5 \mathrm{~h}$ versus $7.0 \mathrm{~h}, \mathrm{P}<0.01)$ and patients required several medical visits. ${ }^{17}$ In a prospective study by Salakos et al. ${ }^{18} 800 \mu \mathrm{g}$ doses of vaginal misoprostol were given every 12 hours for a period of 36 hours (total of $2400 \mu \mathrm{g}$ ) and the success rate was $91 \% .{ }^{18}$ However, in their study the gestational age for miscarriage termination was less than our study ( $<9$ weeks versus 13 weeks), a greater total dose was given and their side effects rate was significantly higher than our rate $(93 \%$ versus $22 \%, \mathrm{P}<0.001$ ). Several studies reported the incidence of side effects as $25-90 \%$ while the overall incidence rate in the present study was only $22 \% .{ }^{15-18}$ The need for curettage was high in our study $45 \%$, but was comparable to the rates reported by others $43-62 \%{ }^{16-18}$ Our higher curettage rate may be due to high false positive ultrasound reports, which may have overestimated the need for it.

In summary, administration of $800 \mu \mathrm{g}$ misoprostol vaginally every 24 hours for a maximum of two doses is effective, safe and associated with high patient satisfaction. It can be offered as a valid alternative to surgical evacuation for patients with incomplete or missed miscarriages at $<14$ weeks' gestation.

\section{Conclusion}

In conclusion, vaginal route misoprostol can be used safely to terminate first trimester spontaneous incomplete or missed miscarriages with acceptable rates of success and high patient satisfaction.

\section{Acknowledgments}

None.

\section{Conflicts of interest}

The authors declare there is no conflict of interests.

\section{References}

1. Laferla JJ. Spontaneous abortion. Clin Obstet Gynaecol. 1986;13(1):105114.

2. Nielsen S, Hahlin M. Expectant management of first trimester miscarriage. Lancet. 1995;345(8942):84-86.

3. Moodliar S, Bagratee JS, Moodley J. Medical vs surgical evacuation of first-trimester spontaneous abortion. Int $J$ Gynecol Obstet. 2005;91(1):21-26.

4. Chung T, Leung P, Cheung LP, et al. A medical approach to management of spontaneous abortion using misoprostol. Acta Obstet Gynecol Scand. 1997;76(3):248-251.

5. Ballagh SA, Harris HA, Demasio K. Is curettage needed for uncomplicated incomplete spontaneous abortion? Am J Obstet Gynecol. 1998;179(5):1279-1282.

6. Doyle NM, Jimenez-Flores J, Ramin MR. Medical versus surgical management of missed abortion: an economic analysis. Obstet Gynecol. 2004;103:4 (Supplement) 65.

7. Demetroulis C, saridogan E, Kunde D, et al. A prospective randomized control trial comparing medical and surgical treatment for early pregnancy failure. Hum Reprod. 2001;16(2):365-369.

8. Goldberg AB, Greenberg MB, Darney PD. Misoprostol and pregnancy. $N$ Engl J Med. 2001;344(1):38-47.

9. Ngai SW, Chan YM, Tang OS, et al. Vaginal misoprostol as medical treatment for first trimester spontaneous miscarriage. Hum Reprod. 2001;16(7):1493-1496.

10. Barbosa RM, Arilha M. The Brazilian experience with Cytotec. Stud Fam Plann. 1993;24(4):236-240.

11. Barcelo F, De Paco C, Lopez-Espin J, et al. The management of missed miscarriage in an outpatient setting 800 versus $600 \mu \mathrm{g}$ of vaginal misoprostol. Aust NZJ Obstet Gynecol. 2012;52(1):39-43.

12. Ho PC, Ngai SW, Liu KL, et al. Vaginal misoprostol compared with oral misoprostol in termination of second-trimester pregnancy. Obstet Gynecol. 1997;90(5):735-738.

13. Ngai SW, Tang OS, Ho PC. Randomized comparison of vaginal (200 g every $3 \mathrm{~h}$ ) and oral (400 mcg every $3 \mathrm{~h}$ ) misoprostol when combined with mifepristone in termination of second trimester pregnancy. Hum Reprod. 2000;15:2205-2208.

14. Bebbington MW, Kent N, Lim K, et al. A randomized controlled trial comparing two protocols for the use of misoprostol in midtrimester pregnancy termination. Am J Obstet Gynecol. 2002;187(4):853-857.

15. Gilbert A, Reid R. A randomized trial of oral versus vaginal administration of misoprostol for the purpose of mid-trimester termination of pregnancy. Aust N Z J Obstet Gynaecol. 2001;41(4):407-410.

16. Neilson JP, Hickey M, Vazquez J. Medical treatment for early fetal death (less than 24 weeks). Cochrane Database. Sys Rev. 2006;3:CD002253.

17. Ngoc NTN, Blum J, Durocher J, et al. A randomized controlled study comparing 600 versus $800 \mathrm{mcg}$ oral misoprostol for medical management of incomplete abortion. Contraception. 2005;72:438-442.

18. Salakos N, Kountouris A, Botsis D, et al. First-trimester pregnancy termination with $800 \mu \mathrm{g}$ of vaginal misoprostol every $12 \mathrm{~h}$. Eur $J$ Contracep Reprod Health Care. 2005;10(4):249-254. 